\title{
SFLASH: Absolute Fluorescence Yield Measurement of Shower Particles.
}

\section{C. H. Jui*}

Dept. of Physics and Astronomy, University of Utah, Salt Lake City, Utah, USA

E-mail: jui@physics.utah.edu

\section{Fukushima}

Institute of Cosmic Ray Research, Tokyo University, Kashiwa, Japan E-mail:

fukushimeicrr.u-tokyo.ac.jp

\section{P. Sokolsky}

Dept. of Physics and Astronomy, University of Utah, Salt Lake City, Utah, USA

E-mail: psephysics.utah.edu

SFLASH is a continuation of the Fluorescence in Air Showers experiment originally run at the SLAC National Laboratory in 2003-2004. SFLASH is designed to measure the absolute fluorescence yield in a developed electromagnetic shower induced by a bunch of about a billion electrons in 1-3 radiation lengths of alumina. These measurements provide a true calibration for fluorescence detectors, such as those of the Telescope Array (TA) experiment, measuring the energies of ultrahigh energy cosmic rays via extensive air showers. This is because the induced shower gives a better representation of the energy distributions particles producing the light in a real extensive air shower than that from mono-energetic beam pulses. A relative measurement was performed in 2004 by FLASH. But because of the high background environment of the Final Focus Test Beam (FFTB) an absolute measurement could not be made. Now, with the availability of End Station A where we could place the photo-multiplier tubes much further, about 10 meters, from the beam, the background has been reduced by more than a factor of 100. Two beam runs were made in July and September of 2017. We will describe the experiment and discuss some preliminary findings.

35th International Cosmic Ray Conference - ICRC2017

10-20 July, 2017

Bexco, Busan, Korea

\footnotetext{
*Speaker.
} 
This is a confirming Abstract for the oral presentation. 